\title{
Formation and removal of alkylthiolate self-assembled monolayers on gold in aqueous solutions
}

\author{
Christie A. Canaria, ${ }^{a}$ Jonathan So, ${ }^{b}$ James R. Maloney, ${ }^{c}$ C. J. Yu, ${ }^{b}$ Jeffrey O. Smith, ${ }^{b}$ Michael L. Roukes, ${ }^{c}$ \\ Scott E. Fraser ${ }^{b}$ and Rusty Lansford ${ }^{* b}$
}

Received 26th July 2005, Accepted 6th December 2005

First published as an Advance Article on the web 3rd January 2006

DOI: $10.1039 / \mathrm{b510661c}$

We report the development of novel reagents and approaches for generating recyclable biosensors. The use of aqueous media for the formation of protein binding alkylthiolate monolayers on $\mathrm{Au}$ surfaces results in accelerated alkylthiolate monolayer formation and improvement in monolayer integrity as visualized by fluorescence microscopy and CV techniques. We have also developed an electrocleaning protocol that is compatible with microfluidics devices, and this technique serves as an on-chip method for cleaning Au substrates both before and after monolayer formation. The techniques for the formation and dissociation of biotinylated SAMs from aqueous solvents reported here may be applied towards the development of Au-based sensor devices and microfluidics chips in the future. A potential use of these devices includes the specific capture and triggered release of target cells, proteins, or small molecules from liquid samples.

\section{Introduction}

An understanding of the interactions of biological molecules with solid supports is vital for the development of detection systems and assay platforms. These relationships are frequently quite complex, involving hydrophobic interactions, electrostatic interactions, van der Waals forces and covalent chemical bonds. We can exploit these interactions in a solid support device by modifying the surface substrate with thin films and monolayers. ${ }^{1}$ Self-assembled monolayers (SAMs) can be engineered to exhibit a variety of chemical properties and reactivities making them hydrophobic, ${ }^{2-4}$ repulsive, ${ }^{5,6}$ or electrochemically active. $^{7-10}$ In practice, SAMs employing biotin have been studied in association with streptavidin conjugated to DNA, proteins, and nanoparticles. ${ }^{1-16}$ Such SAMs are vital for bioassay technologies such as DNA chips, protein chips, and small molecule biosensors.

Alkylthiols form SAMs on Au substrates in distinct stages. ${ }^{17}$ An n-alkylthiol, such as the ones used in this paper, is structurally comprised of an n-alkane chain with a thiol group at one end and a functional group of choice at the other end. We employ triethylene glycol and biotin in the alkylthiols studied here. Upon adsorption, alkylthiols are reduced to alkylthiolates ${ }^{18,19}$ that initially adsorb onto a Au surface in a disordered fashion, resulting in $80-90 \%$ coverage of the substrate. ${ }^{17}$ The subsequent adsorption stage is slower as the alkylthiolates selfassemble into a more organized and insulated film. ${ }^{20}$ Variables such as temperature, thiol concentration, terminating end

${ }^{a}$ Department of Chemistry, California Institute of Technology, 1200 E. California Blvd., Pasadena, CA 91125, USA

${ }^{b}$ Department of Biology, California Institute of Technology, 1200 E.

California Blvd., Pasadena, CA 91125, USA.

E-mail: rusty@gg.caltech.edu; Fax: +1-626-584-9527;

Tel: +1-626-395-2004

${ }^{c}$ Department of Physics, California Institute of Technology, 1200 E.

California Blvd., Pasadena, CA 91125, USA group, ${ }^{21}$ and solvent composition ${ }^{2}$ can affect alkylthiolate monolayer formation on $\mathrm{Au}$.

The organic solvents ethanol, DMSO, or hexane are often used to solvate hydrophobic alkylthiols, ${ }^{22}$ but they are not compatible with polydimethylsiloxane (PDMS), one of the most common materials used for making microfluidic devices (GE Silicones, Electronic Materials Handbook). Depending on the volume of liquid and thickness of the PDMS microfluidics layer, these organic solvents may swell the $\mathrm{PDMS}^{23}$ causing delamination from the Au surface. Evaporation of solvents via the PDMS can also occur and deleteriously effect formation of well-assembled alkylthiol monolayers. Alkylthiols with large hydrophilic groups such as an oligoethylene glycol or an oligonucleotide are soluble in aqueous solvents ${ }^{13}$ that are compatible with PDMS.

SAMs on Au have been successfully made and stored in aqueous solvents. ${ }^{24}$ Monolayers of hydrophobic alkythiol monolayers may be formed in aqueous solutions by the aid of surfactants. ${ }^{24}$ In water, hydrophobic alkythiols spontaneously coordinate to the water-air interface. The addition of surfactants results in micelle formation around alkylthiols and aids in their diffusion to the gold surface. ${ }^{24}$ Studies by Yang, et al. indicate that alkylthiol based SAMs desorb more slowly when stored in water $(5 \%$ DMF or DMSO) compared to butan-2-ol or hexane. ${ }^{25}$ The dilute amphiphilic DMF or DMSO are thought to coordinate to small defect sites to prevent both oxidation and re-solvation of alkanethiolates. Samples undergoing a short thiolate adsorption time followed by incubation in water have also been shown to exhibit more crystalline packing of alkylthiolate chains. ${ }^{26}$ The long alkyl chains associate via van der Waals and hydrophobic interactions in aqueous solutions that promote organization of the stable SAMs on the Au surface. ${ }^{26}$

An essential requirement for high quality alkylthiolate monolayers is a clean $\mathrm{Au}$ substrate. ${ }^{27}$ Clean $\mathrm{Au}$ substrates exposed to ambient conditions will quickly collect impurities 
from the environment and atmosphere that can impact SAM growth. ${ }^{4,28-30}$ These contaminants are thought to be hydrocarbon pollutants that can be cleaned off the Au substrates using strong oxidizing agents such as aqua regia solution, piranha solution, and ozone plasma. ${ }^{22}$ While each of these methods yields clean Au surfaces that permit quality SAM formation, they each present major drawbacks for cleaning integrated biosensors. The thin $\mathrm{Au}$ components within a device, including the test substrates and the electrical leads, are quickly dissolved upon exposure to these acid based cleaning reagents. Ozone plasma effectively oxidizes and degrades Au bound contaminants, but it is difficult to ozone clean the $\mathrm{Au}$ surfaces packaged under PDMS layers.

Other methods such as ozone laser ablation, plasma treatment, and electrochemical cleaning were also considered as options for cleaning $\mathrm{Au}$ pads. However, as test substrates become more densely populated on a chip, laser cleaning of chips becomes limited by wavelength diffraction. As biosensing surfaces shrink to the nano-scale, the small features will need to be addressed using different techniques. The use of nanoelectrodes may be the solution. Electrochemical techniques have been previously reported to clean Au chip surfaces of contaminants effectively enough for alkanethiol attachment and subsequent SAM formation. ${ }^{18,31-35}$ Application of sufficiently oxidative $\left(+0.85 \mathrm{~V}\right.$ vs. $\mathrm{SCE}$ in $\left.0.1 \mathrm{M} \mathrm{H}_{2} \mathrm{SO}_{4}\right)$ or reductive $(-1.03 \mathrm{~V}$ vs. SCE in $0.1 \mathrm{M} \mathrm{NaOH})$ potentials on gold induces desorption of surface species, including the alkylthiolates used to make specific monolayers. ${ }^{36}$ This technique has the additional positive attribute for our studies in that it permits $\mathrm{Au}$ pads at the tips of our cantilevers to be cleaned individually or as a group. Another advantage of electrocleaning is that we can specifically target desired Au pads located at the ends of cantilevers with nanometer resolution, which is essential for the longterm goal of addressing individual cantilevers on a BioNEMS chip.

Here we report the development of a reusable, microfluid based biosensor system by the formation of functionalized alkylthiolate monolayers on addressable Au surfaces. We adsorbed two ethylene glycol modified alkylthiolates (TEG and BAT) (Fig. 1) onto Au surfaces in order to bind specific analytes while repelling non-specific ones. Alkylthiolates containing ethylene glycol reduce the non-specific binding of proteins, bacteria, and cells to $\mathrm{Au}$ and $\mathrm{Si}$ surfaces. ${ }^{37-46} \mathrm{We}$ show that insulating TEG and BAT monolayers on $\mathrm{Au}$ surfaces were adsorbed from water at faster rates and with fewer monolayer pinholes than SAMs adsorbed from ethanol. Finally, electrochemical techniques efficiently remove contaminants from Au surfaces to enhance alkylthiolate monolayer formation in a manner that is addressable on a

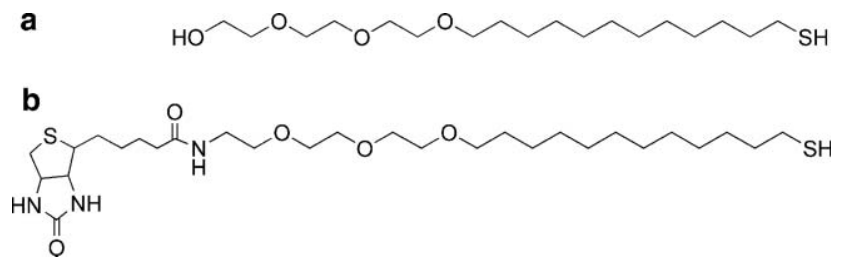

Fig. 1 (a) Tri(ethylene glycol) dodecylthiol (TEG). (b) Biotinylated tri(ethylene glycol) dodecylthiol (BAT). nanometer scale and does not damage the Au surfaces during repeated cleaning.

\section{Materials and methods}

\section{Reagents}

Silicon wafers were purchased from Wafer World. Chromium was purchased from R.D. Mathis Company and gold shots from Refining Systems, Inc. Phosphate buffered saline (PBS) was prepared as $0.139 \mathrm{M} \mathrm{NaCl}, 2.68 \mathrm{mM} \mathrm{KCl}, 8.1 \mathrm{mM}$ $\mathrm{Na}_{2} \mathrm{HPO}_{4}$, and $1.1 \mathrm{mM} \mathrm{K} \mathrm{HPO}_{4}$ (Mallinckrodt) in Nanopure water. Potassium ferrocyanide and potassium ferricyanide were purchased from Aldrich. Absolute ethanol was purchased from Aaper Alcohol and Chemical Company. Cy3 labeled streptavidin was purchased from Zymed, Inc. Reagents BAT and TEG were synthesized in house using techniques disclosed elsewhere. $^{47}$

\section{Preparation of substrates and monolayers}

Silicon wafers were photo-patterned using dark field transparency masks (Fig. 2a) and a positive photoresist. A $3 \mathrm{~nm}$ chromium adhesion layer and $100 \mathrm{~nm}$ Au layer were then thermally evaporated. Diced Au substrates were then plasma cleaned at an oxygen flow rate of $0.8 \mathrm{~L} \mathrm{~min}^{-1}$ in an UV ozone cleaner (SAMCO UV \& Ozone Dry Stripper, Model UV-1) at $100{ }^{\circ} \mathrm{C}$ for $30 \mathrm{~min}$ followed by a $2 \mathrm{~min}$ nitrogen purge. Au substrates used in electrochemical experiments underwent a pre-cleaning treatment by $\mathrm{CV}$ scans out to $1.2 \mathrm{~V}$ (vs. $\mathrm{Ag} / \mathrm{AgCl}$, saturated $\mathrm{KCl}$ ), at a scan rate of $100 \mathrm{mV} \mathrm{s}^{-1}$ in $30 \mathrm{mM}$ ferrocyanide/PBS. These electrocleaned samples were then rinsed in copious amounts of water and ethanol, dried under a stream of argon, and immediately placed in thiol solution. Reagent compositions (BAT and TEG stock solutions of $10 \mathrm{mM}$ in ethanol) were varied for a total thiol concentration of $0.1 \mathrm{mM}$. Diluting the ethanolic stock solution of thiols in absolute ethanol and Nanopure water gave aqueous solvent compositions of $50 \%$ and $1 \%$ ethanol. For brevity, we refer to

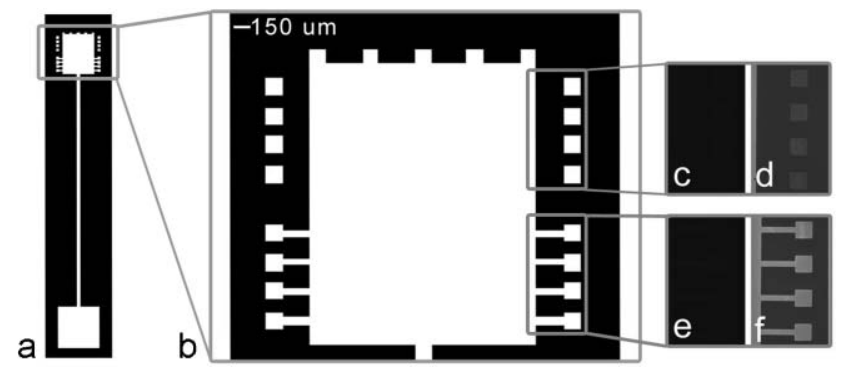

Fig. 2 (a) Photolithography mask used for printing Au electrodes. The bottom square provides ohmic contact for the electrode and the top detailed half serves as the working electrode surface. (b) A magnified view of the working electrode area. Both electrically addressable and isolated $\mathrm{Au}$ pads are presented. Control substrates without SAMs do not bind Cy3-streptavidin; electrochemically untreated sample is (c) and treated is (e). Biotinylated SAMs on electrochemically precleaned $\mathrm{Au}$ bind $\mathrm{Cy} 3$-streptavidin; electrochemically untreated sample is (d) and treated is (f). The relative fluorescence intensities (mean and standard deviation) for Au pads are as follows (4 samples): 2c) $7.8 \pm 0.3,2 \mathrm{~d}) 59.7 \pm 1.9,2 \mathrm{e}) 8.0 \pm 0.1,2 \mathrm{f}) 120 \pm 4$. 
$1 \%$ ethanol in the context of thiol solvent as "water". Upon removal, samples were rinsed in ethanol and dried under argon.

\section{Electrochemical methods}

The structural integrity of the adsorbed monolayer is characterized using CV method. ${ }^{48}$ During a CV scan, a tightly assembled monolayer insulates the Au surface against electron transfer with a redox-active molecule in solution. Any defects in the monolayer film are detected by $\mathrm{CV}$ and characterized by current flow. $\mathrm{CV}$ measurements were carried out with a $\mathrm{CH}$ Instruments Model 600B potentiostat ( $\mathrm{CH}$ Instruments, Austin, TX.) A conventional three-electrode electrochemical cell was constructed with a platinum wire/mesh counter electrode and $\mathrm{Ag} / \mathrm{AgCl}$ reference electrode in saturated $\mathrm{KCl}$. The Au substrate served as the working electrode. Measurements were taken in an electrolyte solution of $30 \mathrm{mM}$ potassium ferrocyanide in PBS at a scan rate of $100 \mathrm{mV} \mathrm{s}^{-1}$. $\mathrm{CV}$ potentials were scanned from $-0.2 \mathrm{~V}$ to $0.8 \mathrm{~V}$. Electrocleaning of samples was accomplished by a CV scan from $-0.2 \mathrm{~V}$ to $1.2 \mathrm{~V}$ at $100 \mathrm{mV} \mathrm{s}^{-1}$ in the same electrolyte solution. Oxidative desorption of SAMs from $\mathrm{Au}$ was accomplished by the application of a 30 second dc pulse at $1.4 \mathrm{~V}$ in an electrolyte solution of PBS.

To qualitatively compare different samples, we calculated peak current densities (PCD) acquired by CV. PCD values for a given sample were determined as the average value for cathodic and anodic current magnitudes for the sample divided by the average value for cathodic and anodic current magnitudes for an untreated, clean gold sample. For clarity, the determination of PCD is given below in eqn (1) where $I_{\mathrm{p}}$ is the current peak height at the anodic or cathodic curves.

$$
\mathrm{PCD}=\frac{\left|\frac{I_{\mathrm{p}_{\text {anodic,sample }}}+I_{\mathrm{p}_{\text {cathodic,sample }}}}{2}\right|}{\left|\frac{I_{\mathrm{p}_{\text {anodic,clean }}}+I_{\mathrm{p}_{\text {cathodic,clean }}}}{2}\right|}
$$

A PCD value of 1 would indicate no monolayer coverage across the Au surface, whereas a PCD value of 0 would indicate complete monolayer coverage across the Au surface. The peak current densities for $\mathrm{CV}$ traces are calculated for each sample condition and are given in Table 1.

Table 1 Calculated peak current density for gold electrodes insulated with BAT and/or TEG alkylthiolates $(0.1 \mathrm{mM}$ final concentration after $1 \mathrm{~h})^{a}$

\begin{tabular}{|c|c|c|c|c|}
\hline \multicolumn{2}{|c|}{ Alkylthiolate percentage } & \multicolumn{3}{|c|}{ Solvent } \\
\hline BAT & TEG & $\mathrm{H}_{2} \mathrm{O}$ & $\mathrm{H}_{2} \mathrm{O} / \mathrm{EtOH}(1: 1)$ & $\mathrm{EtOH}$ \\
\hline 0 & 0 & 1 & 1 & 1 \\
\hline 0 & 100 & 0.00 & 0.43 & 0.31 \\
\hline 100 & 0 & 0.90 & 0.51 & 0.54 \\
\hline 50 & 50 & 0.00 & 0.64 & 0.75 \\
\hline 25 & 75 & 0.02 & 0.49 & 0.78 \\
\hline 12.5 & 87.5 & 0.00 & 0.45 & 0.78 \\
\hline 6.25 & 93.75 & 0.00 & 0.32 & 0.73 \\
\hline
\end{tabular}

\section{Protein-binding assays}

The Au samples with adsorbed BAT/TEG SAMs were submersed in a $200 \mathrm{nM}$ solution of Cy3-labeled streptavidin in PBS for 30 minutes at room temperature. The samples were removed and washed five times with $1 \mathrm{ml}$ of PBS, then stored in PBS for immediate analysis by fluorescence microscopy.

\section{Fluorescence microscopy}

Images were acquired on an upright Zeiss Axioplan 2 infinity corrected microscope (Zeiss, Germany) and acquired with a monochrome CCD Zeiss Axiocam HRm camera. Zeiss PlanNeofluar objectives $10 \times / 0.3,20 \times / \mathrm{NA} 0.5$, and $40 \times / \mathrm{NA} 0.75$ were used in conjunction with a Chroma (Rockingham, VT) Cy3 filter set. A mercury arc lamp served as the excitation source. Images were acquired in 8-bit monochrome resolution and $1030 \times 1300$ pixel resolution.

\section{Results and discussion}

\section{Electrochemical cleaning of Au surfaces improves monolayer coverage}

The cleanliness of substrates and method of cleaning directly influence the quality of alkylthiolate monolayer formation; ${ }^{27}$ the presence of contaminants impedes alkylthiolate monolayer formation. ${ }^{2}$ Contaminants such as hydrocarbons may arise from the environment and adsorb non-specifically to Au surfaces ${ }^{49}$ making cleaning a necessary step prior to alkylthiolate monolayer formation.

To determine how clean an Au substrate is, we can use the conductive properties of $\mathrm{Au}$ to allow cyclic voltammetry (CV) inspection of the quality of the alkylthiolate monolayer coverage on Au surface. CV analysis detects defects in alkylthiolate monolayers, including pinholes, gaps, islands, and disordered packing in general, which can expose the Au substrate to the surrounding solvents. ${ }^{50} \mathrm{Fe}^{2+/ 3+}$ ions are able to exchange electrons with the naked Au substrate or one that is covered by a defective alkylthiolate monolayer, resulting in current flow. Adsorption of $0.1 \mathrm{mM}$ TEG in ethanol reagents after 12 hours at room temperature onto cleaned $\mathrm{Au}$ substrates results in complete monolayer coverage and thus Au insulation from the $\mathrm{Fe}^{2+/ 3+}$ ions in solution (Table 1).

Our Au/Si based chips are made in a Nanofabrication room and are initially cleaned with ozone plasma. The $\mathrm{Au}$ and $\mathrm{Si}$ surfaces of these biosensor chips are receptive to quality alkylthiolate monolayer formation if used immediately (data not shown). However, we regularly make numerous potential biosensor chips at a time and then store them under $\mathrm{N}_{2}$ conditions for periods of days to weeks before use. During this storage time, it is common for unidentified contaminants that deleteriously effect alkylthiolate monolayer formation as determined by $\mathrm{CV}$ analysis to absorb onto the Au surfaces. We observe similar Au surface contamination when biosensor chips are stored overnight in air, 100\% ethanol, 100\% methanol, or ddH20 (data not shown). Contamination of the Au surfaces severely limits our ability to consistently generate quality alkylthiolate monolayers.

We attempted numerous procedures to clean the Au surfaces of stored chips with rinses in ethanol, methanol, isopropanol, 
or acetone, but $\mathrm{CV}$ analysis indicated that the ensuing alkylthiolate monolayer coverage was substandard, suggesting contaminants remained on the Au substrates. More stringent cleaning protocols such as piranha or aqua regis treatments effectively reconditioned the chips to their pre-stored quality. However, the strong acid treatments are highly corrosive to our chips. Specifically, piranha treatment often causes delamination of the $\mathrm{Au}$ from the $\mathrm{Si}$ substrate and destroys the small photo-patterned $\mathrm{Au}$ surface features on our chips (data not shown). We also considered using ozone plasma treatment to recondition the stored chip $\mathrm{Au}$ surfaces as previously mentioned. However, plasma ozone, piranha and aqua regis treatments are not amenable for use with PDMS covered chips due to PDMS compatibility issues or lack of $\mathrm{Au}$ surface accessibility.

Electrocleaning of the $\mathrm{Au}$ substrates can remove bound contaminants without destroying the underlying Au surfaces. ${ }^{51}$ Electrocleaning also permits the sequential or simultaneous cleaning of the numerous Au substrates located throughout our biosensor chips. The efficacy of electrochemical treatments on Au electrodes and subsequent SAM formation were tested by comparing electrically connected $\mathrm{Au}$ substrates against electrically isolated Au substrates on a single chip (Fig. 2a). Au regions that were electrically connected were cleaned during the anodic potential sweep to $+1.2 \mathrm{~V}\left(100 \mathrm{mV} \mathrm{s}^{-1}\right)$, whereas electrically isolated Au regions were not. The entire chip was then immersed in a BAT:TEG containing ethanol solution in order to form at BAT:TEG monolayer. The chips were then incubated with streptavidin-Cy3 in order to indirectly assay the quality of alkylthiolate monolayer formation since $\mathrm{CV}$ analysis was not feasible on the electrically isolated $\mathrm{Au}$ substrates. The relative fluorescent intensity of the streptavidin-Cy3 protein binding to the BAT:TEG monolayer was determined using fluorescent microscopy. For these samples, the electrically isolated regions consistently exhibited half the fluorescence intensity compared to the electrically connected regions (Fig. 2c-f). These data are interpreted to suggest that pre-cleaned $\mathrm{Au}$ substrates are able to specifically bind more protein since they can bind more biotin on the higher quality BAT:TEG mixed monolayers on cleaned Au substrates compared to the inferior BAT:TEG absorption on dirty $\mathrm{Au}$ substrates.

\section{Effect of adsorption solvent on SAM formation}

Certain organic solvents (i.e. DMSO, DMF, hexane) that are used to generate alkylthiolate monolayers are not compatible with PDMS or biological agents ${ }^{23}$ (GE Silicones, Electronic Materials Handbook). We explored the possibility of generating alkylthiolate monolayers using the solvents ethanol and/or water. We first tested the solubility of BAT and TEG in ethanol and water based solvents and then determined their ability to form quality alkylthiolate monolayers. We used CV to evaluate the quality of the alkylthiolate monolayer coverage of Au substrates and protein binding to evaluate the biological functionality of the formed alkylthiolate monolayers.

The solubility of TEG, BAT, and BAT:TEG mixtures in water and ethanol solutions varies. At $0.1 \mathrm{mM}$ thiol concentrations, TEG dissolves completely in both water and ethanol.
However, BAT and BAT:TEG solutions (1:4) yield a white precipitate in solvent compositions lower than $30 \%$ ethanol. At higher ethanol concentrations, no precipitate was observed. We chose $50 \%$ ethanol as the intermediate solvent composition in our study because the effects of equivalent amounts of water and ethanol could be observed, and there would be no interference in SAM formation due to insoluble thiol molecules. Solubility of the thiol molecule in a given solvent plays a key role in the formation of SAMs ${ }^{52-54}$ Based on our combined observations of thiol solubility, our CV measurements, and fluorescence microscopy data we can infer the composition and extent of SAM formation on Au substrates in our aqueous solutions.

Of all the thiol compositions tested here, pure TEG is the most soluble in ethanolic and aqueous solvents. Solutions of pure TEG form the most insulating SAMs and form SAMs at the fastest rate. The TEG molecule has two distinct parts: a twelve carbon hydrophobic alkyl chain and a hydrophilic triethylene glycol chain (Fig. 1a). From Table 1, the peak current density value for TEG-coated electrodes was lowest when SAMs were adsorbed from water $(<0.001)$, indicating a highly insulating monolayer. In comparison, TEG SAMs formed in 50\% ethanol and ethanol have higher PCD values, 0.43 and 0.31 , respectively. There exist unfavorable interactions between water and the alkylthiol chain of TEG and attractive hydrophobic interactions between the alkylthiol chains. ${ }^{55}$ An energetic penalty would be imposed for any SAM defect that increases the amount of the hydrophobic alkyl chains exposed to water. Therefore, SAMs prepared in aqueous solutions are likely more well-ordered with fewer defects than those prepared from ethanol solutions. ${ }^{24}$ Over the course of 24 hours, the TEG SAMs in 50\% ethanol and ethanol also form highly insulating monolayers, but that time scale is an order of magnitude larger than for TEG SAMs formed in water.

BAT is soluble in ethanol, but is only partially soluble in aqueous solvents. BAT is similar to TEG, but also includes a hydrophobic biotin group (Fig. 1b). At $0.1 \mathrm{mM}$, BAT forms a white precipitate when diluted in water or in ethanolic solvents of $30 \%$ ethanol and lower. We observe from Table 1 PCD values that BAT forms SAMs with less defects when adsorbed from ethanolic solutions than when adsorbed from water. In water, the insoluble nature of BAT likely makes delivery of the molecule to the liquid/Au interface more difficult. Only after longer incubation times ( $>24 \mathrm{~h}$ ) do BAT molecules adsorbed from water make insulating monolayers (data not shown). The pure BAT SAMs formed from ethanolic solutions only provide partial coverage and insulation across the Au surface after a 1 hour incubation at RT (Table 1). The lack of full electrode insulation may be due to steric interference of the BAT biotin end groups. The triethylene glycol group between the alkane chain and the biotin group is long enough to allow the hydrophobic biotin to wrap around and bury into the hydrophobic alkane chains. ${ }^{56}$ This likely prevents the tight association of the alkane regions of the alkylthiolates required for the formation of tightly packed, defect-free monolayers. In a previous study, angle-resolved X-ray photoelectron spectroscopy revealed that biotin head groups of BAT molecules were buried within the alkyl chain monolayer. ${ }^{56}$ In contrast to pure TEG solutions 
that form higher quality SAMs in water, pure BAT solutions form higher quality SAMs in ethanolic solvents.

Similar to pure BAT solutions, mixed BAT:TEG solutions exhibited partial solubility in ethanolic solvents of $30 \%$ and below. The mixed SAMs tested had BAT percentage compositions ranging from $50 \%$ to $6.25 \%$. From Table 1 , the general trend of PCD values measured for these mixed monolayers indicates a decrease in PCD, or increase in monolayer quality, as aqueous composition of solvent increases and as TEG composition of the thiol mixture increases. We postulated above that TEG monolayer formation is greatly influenced by the presence of water. It would appear that the hydrophobic interactions of the alkyl chains in both BAT and TEG coupled with an energetic penalty of defects within a monolayer are also a dominant force in the formation of mixed monolayers. Mixed monolayers formed from ethanol also indicate that SAM quality increases as TEG composition increases. We show that incorporation of TEG into a BAT monolayer can increase the order of the alkyl chains within the monolayer. The ethylene glycol groups of TEG can coordinate with the ethylene glycol groups of BAT, reducing the likelihood of biotin groups burying into the alkane chains of the monolayer. Biotin groups that might otherwise be buried within the monolayer are presented to the solvent interface and thus are accessible for binding events.

\section{Specific binding of fluorescent proteins to SAMs}

In order to assay the composition and functionality of the BAT:TEG mixed alkylthiolate monolayers, we used fluorescence microscopy and $\mathrm{CV}$ on the same $\mathrm{Au}$ substrates to correlate the relative fluorescence intensities of Cy3-labeled streptavidin with the amount of accessible BAT molecules on the Au substrates. Au chip substrates were immersed in mixed thiol solutions $([\mathrm{BAT}]+[\mathrm{TEG}]=0.1 \mathrm{mM})$ of 3 different solvent compositions (100\% ethanol, 50\% ethanol, and water) for 60 minutes at room temperature and subsequently incubated with $200 \mathrm{mM}$ Cy3-labeled streptavidin in PBS for 30 minutes at room temperature. Based on the protein-binding assay, fluorescence levels for Au samples linearly increased as the BAT concentration of the thiol solutions increased (Fig. 3). However, thiol composition in solution does not necessarily correlate with the thiol composition of the adsorbed SAM. ${ }^{56}$ For a given mixed thiol composition, mixed monolayers adsorbed from water have more thiol molecules on $\mathrm{Au}$ than monolayers adsorbed from ethanol, as indicated by CV data; however, the number of BAT molecules adsorbed are comparable, as indicated by fluorescence measurement. From this, we deduce that the TEG composition of monolayers adsorbed from water is higher versus monolayers adsorbed from ethanol. Formation of mixed SAMs from 50\% ethanol is governed by the solubility of TEG and BAT in the solvent. PCD values for SAMs formed from $50 \%$ ethanol indicate intermediate monolayer coverage compared to water and ethanol conditions. Solubility of BAT in $50 \%$ ethanol is assisted by the presence of TEG. We expect the surface ratio of BAT/TEG to be lower compared to monolayers formed from ethanol, since multiple TEG can coordinate around a single BAT. As the thiols approach the Au surface, there is a higher local concentration of TEG to BAT. These result in fewer BAT incorporated into the monolayer and lower levels of Cy3-protein adsorption. The decrease in protein binding by monolayers adsorbed from aqueous ethanol solution may be attributed in part to the observed solubility properties of the BAT and TEG reagents.

\section{Electrocleaning to recycle Au substrates}

We have explored the utility of using electrochemical methods for the directed or triggered release of alkylthiolate monolayers from $\mathrm{Au}$ in order to regenerate the $\mathrm{Au}$ surface for the subsequent and repeated formation of new alkylthiolate monolayers. We chose to focus on electrochemical methods of alkylthiolate monolayer desorption because of the control, speed and amenability of the protocol to arrays of electrodes in

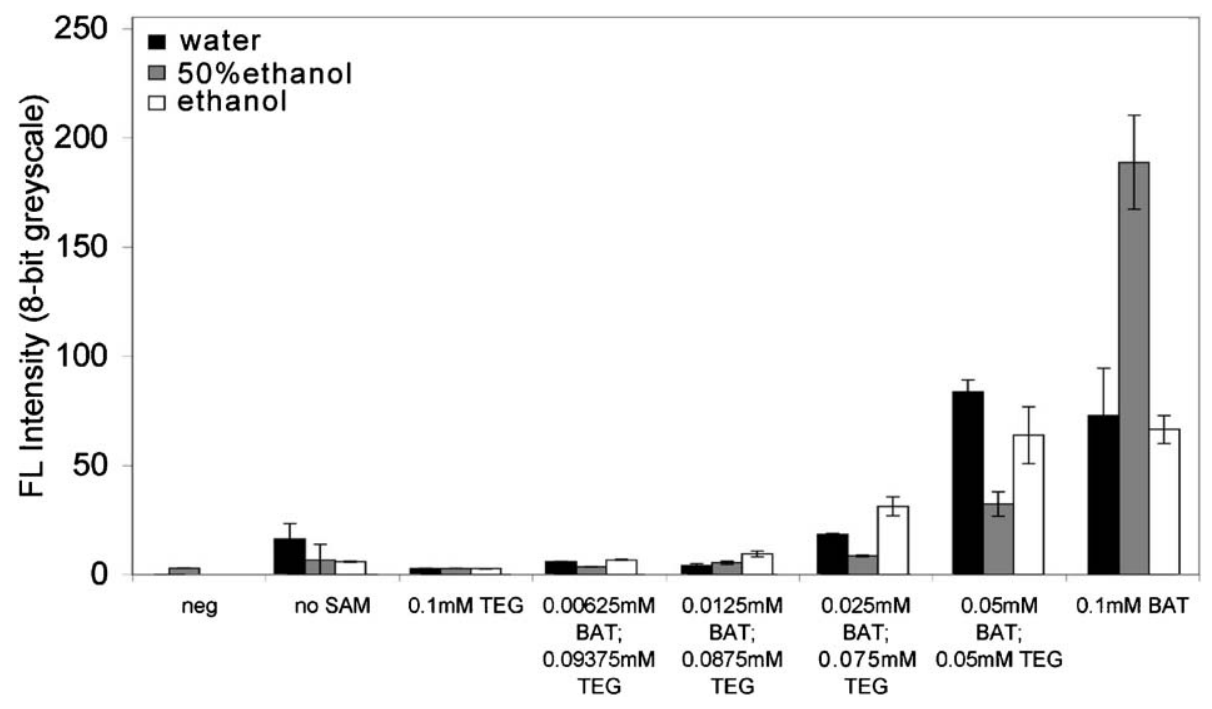

Fig. 3 Relative fluorescence intensities plotted in a bar graph displaying signal for Au samples incubated in Cy3-streptavidin under each thiol adsorption condition. Thiols were adsorbed for 1 hour prior to Cy3-streptavidin incubation. Each graph represents the average of 16 independent samples 




(f)

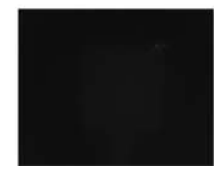



(g)

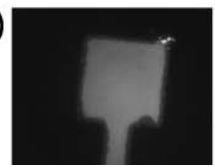



(h)

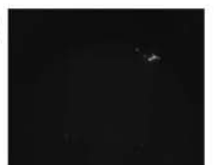

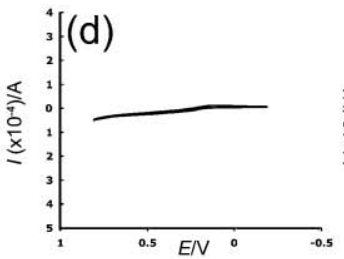

(i)

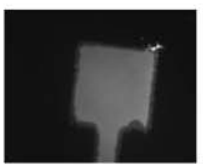

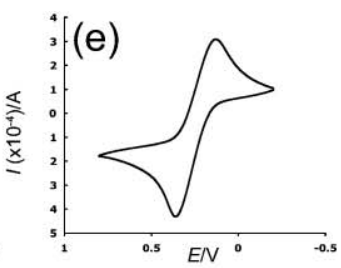

(j)

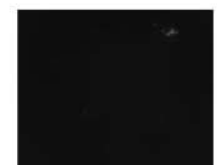

Fig. $4 \mathrm{CV}$ traces and corresponding fluorescence microscopy images for Au electrode as it undergoes re-cycling treatments. Two cycles are shown. (a), (f) Bare Au electrode. (b), (g) After $1 \mathrm{~h}$ of BAT:TEG (1:4) SAM formation. (c), (h) After $30 \mathrm{~s}$ dc pulse at $1.4 \mathrm{~V}(v s . \mathrm{Ag} / \mathrm{AgCl}, \mathrm{saturated} \mathrm{KCl)}$. (d), (i) After second BAT:TEG (1:4) SAM formation. (e) After $30 \mathrm{~s}$ dc pulse at $1.4 \mathrm{~V}$ (vs. Ag/AgCl, saturated $\mathrm{KCl}$ ).

microfluidic devices. Thermal desorption, displacement of short chain alkylthiolates by longer chain alkylthiolates, piranha solutions, and plasma oxidation are all applications that work well for cleaning bulk samples, but not for addressing single Au samples within a sensor array. Metal polishing and piranha solution are damaging to small metal features.

Electrochemical desorption of alkylthiolate monolayers, however, allows us to control individual electrodes, is compatible with PDMS-based microfluidics systems and has the added advantage of being rapid. The application of both reductive $^{34,57,58}$ and oxidative ${ }^{36,58}$ potentials for the desorption of alkylthiolate monolayers from Au have been reported.

Our attempts to reductively desorb alkylthiolate monolayers from $\mathrm{Au}(-1.0 \mathrm{~V} v s$. $\mathrm{Ag} / \mathrm{AgCl})$ resulted in the desorption of alkylthiolate monolayers along with the delamination of the $\mathrm{Au}$ from the Si substrate, which is consistent with previous reports. ${ }^{51} \mathrm{We}$ tested the application of $\mathrm{dc}$ and ac anodic potentials $(0.8 \mathrm{~V}$ to $1.4 \mathrm{~V})$ at various time pulses $(2-60 \mathrm{~s})$ to determine an optimized alkylthiolate monolayer removal protocol. The ac anodic potentials were additionally tested at frequencies ranging from $10 \mathrm{~Hz}-10 \mathrm{kHz}$. CV measurements were taken to characterize the surface coverage of alkylthiolate monolayers on Au surfaces treated under the different cleaning protocols (data not shown). We found that the shortest, most effective dc cleaning protocol was a $30 \mathrm{~s}$ pulse at $1.4 \mathrm{~V}$. The most effective ac treatment was an ac potential of $1.2 \mathrm{~V}$ with an amplitude of $0.2 \mathrm{~V}$ at a frequency of $1 \mathrm{kHz}$ for $30 \mathrm{~s}$.

We next investigated whether the chip Au substrates could withstand multiple alkylthiolate monolayer formation-anddesorption cycles. Using both $\mathrm{CV}$ and fluorescence microscopy, Au substrates were characterized throughout the process which included the following steps (Fig. 4): (1) $\mathrm{Au}$ surface electrocleaning (anodic sweep to $1.2 \mathrm{~V}$ ); (2) formation of BAT:TEG monolayer (1:4 thiol ratio; $0.1 \mathrm{mM}$ in water for 60 minutes, RT), (3) adsorption of Cy3-strp (30 min, RT), and (4) repeat cycle. For a given chip, CV traces for the pre-cleaned electrode overlap very well with the CV traces for the chip after oxidative desorption of the alkylthiolate monolayer. Traces for the BAT:TEG monolayer yield very low current, indicating quality monolayer formation. Fluorescent images of the precleaned Au (Fig. 4(f)) and post-SAM cleaned Au (Fig. 4(h) and (j)) yielded background fluorescence intensities. The biotinylated SAMs bound Cy3-strp, yielding fluorescence images of Au pads with positive signal. The electrocleaning protocols could be repeated at least ten times without any noticeable blemishes to the Au substrates.

We demonstrate the ability to repeatedly form and remove alkylthiolate monolayers on Au surfaces using a method that is compatible with a packaged microfluidics device. The accelerated formation of BAT and TEG alkylthiolate monolayers from water results in low-defect monolayers. These functionalized substrates have potential applications in biosensor devices and for metal substrate passivation. ${ }^{59}$ Additionally, the ability to electrochemically remove alkylthiolate monolayers from the Au surface permits us to recycle our devices at least 10 times allowing capture as well as release of cells or proteins bound via specific monolayers. A complete cycle of Au substrate cleaning, alkylthiolate monolayer formation and alkylthiolate monolayer removal requires 70 minutes, which will likely be important for making fluidic based chips able to assay multiple analytes. This reduction in monolayer formation and cleaning times speeds up the process of creating recyclable biological sensors for lab on a chip experiments.

\section{Acknowledgements}

This research was funded under DARPA grant MLR.00018-3AFOSR.000019 and RDL.NASA.000143. The authors thank Blake Axelrod, Jessica Arlett, and Melaku Muluneh for their assistance in materials fabrication. C.A.C. thanks John Keith and Brad Cenko for helpful discussions.

\section{References}

1 M. Mrksich, Chem. Soc. Rev., 2000, 29, 267-273.

2 C. D. Bain, J. Evall and G. M. Whitesides, J. Am. Chem. Soc., 1989, 111, 7155-7164.

3 C. D. Bain and G. M. Whitesides, J. Am. Chem. Soc., 1989, 111.

4 C. T. Bain, E. B. Troughton, Y. Tao, J. Evall, G. M. Whitesides and R. G. Nuzzo, J. Am. Chem. Soc., 1989, 111, 321-335.

5 C. G. Golander, J. N. Herron, K. Lim, P. Claesson, P. Stenius and J. D. Andrade, Properties of immobilizated peg films and the interaction with proteins, in Poly(ethylene glycol) chemistry, biotechnical and biomedical applications, ed. J. Harris, Plenum Press, New York, 1992, pp. 221-245.

$6 \mathrm{~J}$. Harris, in Poly(ethylene glycol) chemistry, biotechnical and biomedical applications, Plenum Press, New York, 1992.

7 M. Mrksich, L. E. Dike, J. Tien, D. E. Ingber and G. M. Whitesides, Exp. Cell Res., 1997, 235, 305-313.

8 C. D. Hodneland and M. Mrksich, Langmuir, 1997, 13, 6001-6003. 
9 M. M. Yousaf, J. Am. Chem. Soc., 1999, 121, 4286-4287.

10 C. D. Hodneland and M. Mrksich, J. Am. Chem. Soc., 2000, 122, 4235-4236.

11 K. Kukanskis, J. Elkind, J. Melendez, T. Murphy, G. Miller and H. Garner, Anal. Biochem., 1999, 274, 7-17.

12 J. Schumaker-Parry, M. Zareie, R. Aebersold and C. Campbell, Anal. Chem., 2004, 76, 918-929.

13 M. Riepl, K. Enander and B. Liedberg, Langmuir, 2002, 18, 7016-7023

14 A. Zybin, C. Grunwald, V. Mirsky, J. Kuhlmann, O. Wolfbeis and K. Niemas, Anal. Chem., 2005, 77, 2393-2399.

15 G. Zhen, V. Eggli, J. Voros, P. Zammaretti, M. Textor, R. Glcokshuber and E. Kuennemann, Langmuir, 2004, 20, 10464-10473.

16 A. Arakaki, S. Hideshima, T. Nakagawa, D. Niwa, T. Tanaka, T. Matsunaga and T. Osaka, Biotechnol. Bioeng., 2004, 88, 543-546.

17 R. F. DeBono, G. D. Loucks, D. D. Manna and U. J. Krull, Can J. Chem., 1996, 74, 677.

18 C. A. Widrig, C. Chung and M. D. Porter, J. Electroanal. Chem., 1991, 310, 335-359.

19 M. Hasan, D. Bethell and M. Brust, J. Am. Chem. Soc., 2002, 124, $1132-1133$.

20 G. Hahner, C. Woll, M. Buck and M. Grunze, Langmuir, 1993, 9, 1955.

21 G. E. Poirier, E. D. Plyant and J. M. White, J. Chem. Phys., 1996, 104, 7325.

22 J. C. Love, L. A. Estroff, J. K. Kriebel, R. G. Nuzzo and G. M. Whitesides, Chem. Rev., 2005, 1103-1169.

23 J. N. Lee, C. Park and G. M. Whitesides, Anal. Chem., 2003, 75, 6544-6554.

24 D. Yan, J. Jordan, V. Burapatana and G. Jennings, Langmuir, 2003, 19, 3357-3364.

25 G. Yang, N. Amro, Z. Starkewolfe and G.-Y. Lui, Langmuir, 2004, 20, 3995-4003.

26 U. K. Sur and V. Lakshminarayanan, J. Electroanal. Chem., 2004 565, 343-350.

27 Z. Yang, A. Gonzales-Cortes, G. Jourquin, J.-C. Vire, J.-M Kauffmann and J.-L. Delplancke, Biosens. Bioelectron., 1995, 10, 789-795.

28 R. G. Nuzzo, L. H. Dubois and D. L. Allara, J. Am. Chem. Soc., 1990, 112, 558

29 K. A. Peterlinz and R. Georgiadis, Langmuir, 1996, 12, 4731-4740.

30 S. Xu, S. Cruchon-Dupetrat, J. C. Garno, G. Liu, G. Jennings, T. Yong and P. E. Laibinis, J. Chem. Phys., 1998, 108, 5002.

31 C. Zhong, J. Zak and M. D. Porter, J. Electroanal. Chem., 1997, 421, 9-13.

32 T. Kakiuchi, H. Usui, D. Hobara and M. Yamamoto, Langmuir, 2002, 18, 5231-5238.

33 D. F. Yang, C. P. Wilde and M. Morin, Langmuir, 1996, 12, $6570-6577$.
34 X. Jiang, R. Ferrigno, M. Mrksich and G. M. Whitesides, J. Am Chem. Soc., 2003, 125, 2366-2367.

35 J. Shepherd, A. Kell, E. Chung, C. Sinclar, M. Workentin and C. Bizzotto, J. Am. Chem. Soc., 2004, 126, 8329-8335.

36 F. Loglio, M. Schweizer and D. Kolb, Langmuir, 2003, 19, $830-834$.

37 P. Harder, M. Grunze, R. Dahint, G. M. Whitesides and P. E. Laibinis, J. Phys. Chem. B, 1998, 102, 426-436.

38 R. Valiokas, S. Svedhem, S. Svensson and B. Liedberg, Langmuir, 1999, 15, 3390-3394.

39 N. A. Alcantar, E. S. Aydil and J. Israelachvili, J. Biomed. Mater. Res. Part B: Appl. Biomater., 2000, 51, 343-351.

40 Y. Chan, R. Schweiss, C. Werner and M. Grunze, Langmuir, 2003, 19, 7380-7385.

41 R. Wang, H. Kreuzer, M. Grunze and A. Pertsin, Phys. Chem. Chem. Phys., 2000, 2, 1721-1727.

42 K. Feldman, G. Hahner, N. Spencer, P. Harder and M. Grunze, J. Am. Chem. Soc., 1999, 121, 10134-10141.

43 D. Schwendel, R. Dahint, S. Herrwerth, M. Schloerholz, W. Eck and M. Grunze, Langmuir, 2001, 17, 5717-5720.

44 S. Herrwerth, T. Rosendahl, C. Feng, J. Fick, W. Eck, M. Himmelhaus, R. Dahint and M. Grunze, Langmuir, 2003, 19, 1880-1887.

45 S. Herrwerth, W. Eck, S. Reinhardt and M. Grunze, J. Am. Chem. Soc., 2003, 125, 9359-9366.

46 A. Pertsin, M. Grunze and I. Garbuzova, J. Phys. Chem. B, 1998, 102, 4918-4926.

47 C. A. Canaria, J. O. Smith, C. J. Yu, S. E. Fraser and R. Lansford, Tetrahedron Lett., 2005, 46, 4813-4816.

48 M. D. Porter, T. B. Bright, D. L. Allara and C. E. D. Chidsey, J. Am. Chem. Soc., 1987, 109, 3559-3568.

49 M. Amrein and D. Muller, Nanobiology, 1999, 4, 229-256.

50 F. Schreiber, Prog. Surf. Sci., 2000, 65, 151-256.

51 C. Hodneland, PhD, University of Chicago, 2001.

52 T. Ishida, W. Mizutani, H. Azehara, F. Sato, N. Choi, U. Akiba, M. Fujihira and H. Tokumoto, Langmuir, 2001, 17, 7459-7463.

53 T. W. Schneider and D. A. Buttry, J. Am. Chem. Soc., 1993, 115, 12391-12397.

54 R. Yamada, H. Sakai and K. Uosaki, Chem. Lett., 1999, 7, 667-668.

55 D. Hatchett, R. Uibel, K. Stevenson, J. Harris and H. White, J. Am. Chem. Soc., 1998, 120, 1062-1069.

56 K. E. Nelson, L. Gamble, L. S. Jung, M. S. Boeckl, E. Naeemi, S. L. Golledge, T. Sasaki, D. G. Castner, C. T. Campbell and P. S. Stayton, Langmuir, 2001, 17, 2807-2816.

57 M. P. Walczak, D. D. Popenoe, R. S. Deinammer, B. D. Lamp, C. Chung and M. D. Porter, Langmuir, 1991, 7, 2687-2693.

58 D. F. Yang, H. Al-Maznai and M. Morin, J. Phys. Chem. B, 1997, 101, 1158-1166.

59 C. Whelan, M. Kinsella, H. Ho and K. Maex, J. Electron. Mater., 2004, 33, 1005-1011. 